\title{
Skp2 expression has different clinicopathological and prognostic implications in lung adenocarcinoma and squamous cell carcinoma
}

\author{
KAIZE ZHONG ${ }^{1}$, FAN YANG ${ }^{1}$, QIUYING HAN ${ }^{2}$, JING CHEN ${ }^{2}$ and JUN WANG ${ }^{1}$ \\ ${ }^{1}$ Department of Thoracic Surgery, Peking University People's Hospital, Beijing 100044; ${ }^{2}$ State Key Laboratory of Proteomics, \\ Institute of Basic Medical Sciences, National Center of Biomedical Analysis, Beijing 100850, P.R. China
}

Received October 15, 2017; Accepted April 23, 2018

DOI: $10.3892 / \mathrm{ol} .2018 .9000$

\begin{abstract}
High expression of S-phase kinase associated protein 2 (Skp2) is associated with numerous clinicopathological parameters, including histology, lymph node metastasis, smoking status, differentiation and Tumor-Node-Metastasis stage in non-small cell lung cancer (NSCLC). Skp2 protein is overexpressed in lung squamous cell carcinoma (LUSC), compared with lung adenocarcinoma (LUAD), whilst the clinicopathological and prognostic implications in LUAD or LUSC remain unclear. A larger study is required to assess the differences in Skp2 expression between these NSCLC types. In the present study, the clinicopathological features and immunohistochemical expression of the Skp2 protein were studied in 500 patients with NSCLC (351 with LUAD and 149 with LUSC). Survival analyses were performed using Kaplan-Meier method and Cox regression model. Skp2 associated genes were identified based on the data from The Cancer Genome Atlas database. Skp2 was overexpressed in patients with LUSC, compared with LUAD $(\mathrm{P}<0.001)$. In histology subgroup analysis, differences in Skp2 protein expression were observed in patients with LUAD, based on sex, differentiation, smoking history, stage, lymph node metastasis and tumor diameter $(\mathrm{P}<0.05)$, but not in patients with LUSC except for smoking status. High Skp2 protein expression in patients with LUAD was associated with reduced overall survival (OS; $\mathrm{P}<0.001)$, but not in patients with LUSC $(\mathrm{P}=0.686)$. The multivariate analysis demonstrated that $\mathrm{Skp} 2$ expression is an independent unfavorable prognostic factor for OS in patients with LUAD $(\mathrm{RR}=1.845, \mathrm{P}<0.05)$. Bioinformatics analyses revealed that minichromosome maintenance complex component 2 , cell division cycle 45 , replication factor $\mathrm{C}$ subunit 4 ,
\end{abstract}

Correspondence to: Professor Jun Wang, Department of Thoracic Surgery, Peking University People's Hospital, 11 Xizhimen South Street, Beijing 100044, P.R. China

E-mail: wangjunpkuph@sina.com

Key words: S-phase kinase associated protein 2, lung cancer, prognosis, tissue microarrays which are differently expressed in LUAD and LUSC, are associated with Skp2 expression and participate in DNA replication and $\mathrm{G}_{1} / \mathrm{S}$ transition. Skp2 protein expression differs in LUAD and LUSC. The clinicopathological and prognostic implications based on Skp2 expression in LUAD and LUSC should be considered different. LUSC with high Skp2 expression may have robust proliferation ability.

\section{Introduction}

The F-box protein S-phase kinase associated protein 2 (Skp2), a component of the Skp1-Cullin 1-F-box E3 ubiquitin-ligase complex, promotes the degradation of the cyclin-dependent kinase inhibitor p27, a key regulator of $G_{1}$ progression. An inverse correlation between Skp2 and p27 protein level has been reported in tumors $(1,2)$. Under physiological conditions, Skp2 controls the initiation of mitosis in that its expression peaks at the $S$ and $G_{2}$ phases, but not at $G_{0}$ and $G_{1}$ phases $(3,4)$. Skp2 is key in the p27 ubiquitin degradation pathway and may inhibit the proliferation of a variety of cell types through the ubiquitin-proteasome pathway (4-6).

Skp2 overexpression has been observed in various human cancers, including lymphomas (7), prostate cancer (8), melanoma (9), pancreatic cancer (10), gastric cancer (11), breast cancer (12), colorectal cancer (13) and lung cancer $(14,15)$.

The involvement of Skp2 overexpression in metastasis and aggressiveness has been reported in many tumors including melanoma (9), pancreatic cancer (10), breast (12), colorectal cancer (13) and lung cancer (1). Skp2 is also considered to be associated with the occurrence, development and prognosis of malignant tumors such as breast cancer (12), melanoma (9), prostate cancer (8), colorectal cancer (14), and lung cancer (1).

Expression of Skp2 is significantly upregulated in primary non-small cell lung cancer (NSCLC), compared with non-tumorous lung tissues (16). In patients with NSCLC, overexpression of the Skp2 protein is associated with numerous clinicopathological parameters, including histology, lymph node metastasis, smoking status, differentiation and tumor stage $(1,16)$. In addition, the positive expression of Skp2 is correlated with a poor prognosis in patients with NSCLC (1).

Concerning the two major pathological types of NSCLC, Skp2 is notably overexpressed in lung squamous cell carcinoma 
(LUSC), compared with lung adenocarcinoma (LUAD) (1). Due to the limitation of sample amounts, it remains unclear whether Skp2 expression is associated with those clinicopathological features in LUAD and LUSC, respectively; therefore, a study with a greater number of patients and specimens is required to compare the clinicopathological and prognostic implications with the Skp2 expression in these NSCLC types.

In the present study, the correlation between clinicopathological features and Skp2 protein expression was investigated in 351 LUAD and 149 LUSC samples. Additionally, the prognostic value of Skp2 protein expression was also investigated. Bioinformatics analyses were performed to investigate Skp2 expression-associated mRNAs and lung cancer mutations.

\section{Materials and methods}

Cases and specimens. Patients were excluded due to chemotherapy or radiotherapy prior to surgery. A total of 351 patients with LUAD and 149 patients with LUSC who were diagnosed and underwent surgery at Peking University People's Hospital (Beijing, China) between January 2004 and December 2012 were enrolled in the present study. The patients included 305 males and 195 females, with a median age of 64 years (range, 24-86). All procedures performed in the current study involving human participants were in accordance with the ethical standards of the Ethics Committee of Peking University People's Hospital. Informed consent was obtained from all individual participants included in the study. All patients were followed up following surgery at 3-6 month intervals, and the total follow-up periods ranged from 4.8 to 7.6 years, with a median of 5.9 years. Complete clinical information from the clinical and pathological records, including sex, age, tumor size, lymphatic invasion, differentiation grade and pathological histology were collected and summarized in Table I.

Normal tissue was collected from at $>2 \mathrm{~cm}$ away from the primary cancer site and histopathologically identified as normal by a pathologist at the Peking University People's Hospital. Fresh tissue samples from each patient were formalin-fixed $(10 \%)$ at room temperature overnight, paraffin-embedded and constructed into tissue microarrays (TMA).

TMA and immunohistochemistry (IHC). TMA were prepared using carefully identified representative core-tissue specimens ( $2 \mathrm{~mm}$ in diameter) as previously described (17). Sections $4 \mu \mathrm{m}$ thick from the recipient blocks were cut. Antigen retrieval was performed in a pressure cooker, followed by treatment with $3 \%$ hydrogen peroxide for 15 min to block endogenous peroxidase activity. IHC analysis was performed using an antibody against Skp2 at room temperature for $2 \mathrm{~h}$ (1:200 dilution; cat. no. 2652; Cell Signaling Technology, Beverly, MA, USA). The sections were rinsed and incubated for $30 \mathrm{~min}$ with biotinylated second antibody in room temperature. Expression of Skp2 was detected using a diaminobenzidine reaction for $30 \mathrm{~min}$ and hematoxylin counterstaining for $30 \mathrm{sec}$ at room temperature. There were two independent pathologists, who were blind to the patients' clinical data, who scored the staining intensity level as follows: Level-0, negative; level-1, weak positive; level-2, moderate positive; level-3, strong positive, and the percentage of positive cells. Skp2 protein expression assessment was based on the method of a semi-quantitatively scoring system (18). The final score was obtained from multiplying the staining intensity level and the percentage of positively stained cells (A for level-1, B for level-2, C for level-3). The calculation formula was as follows: Score $=1 \times \mathrm{A} \%+2 \mathrm{xB} \%+3 \mathrm{xC} \%$. The semi-quantitative method provided further information regarding the expression of Skp2 and reduced bias, whilst analyzing the correlation with clinicopathological characters.

Skp2 co-expression analysis of LUAD and LUSC differential $m R N A s$. The Cancer Genome Atlas (TCGA) mRNA-expression data for LUSC and LUAD were obtained from Firehose (http://gdac.broadinstitute.org/) using the Bioconductor package RTCGAToolbox (version 2.4.0; http://bioconductor.org/packages/RTCGAToolbox/). For LUAD, a provisional dataset of 162 total tumors was used, and for LUSC, 240 samples were used.

Differential mRNAs were screened using the Bioconductor package edgeR (version 3.16.5; http://bioconductor.org/packages/edgeR/) (19). The FDR threshold was set to 0.05 , in order to identify significantly deviating genes between LUAD and LUSC. The LogFC threshold was set to 1.5 to select mRNAs for the following co-expression analysis. The $\mathrm{R}$ statistical language (version3.4.1; https://www.r-project.org/) was used to conduct these analyses.

The spearman rank correlation method was used to assess the co-expression of the differential mRNAs. The top 50 Skp2-associated mRNAs were cluster analyzed and plotted by R package heatmap (version1.0.8; https://CRAN.R-project. org/package=pheatmap). The highest associated mRNAs were processed in The Database for Annotation, Visualization and Integrated Discovery functional annotation bioinformatics analysis $(20,21)$.

TCGA gene expression and mutation data for LUAD and LUSC were obtained from the cBioPortal for Cancer Genomics (www.cbioportal.org) $(22,23)$. For LUAD, the provisional dataset of 522 total tumors was used. Mutation data were obtained for tumor protein P53 (TP53), KRAS, Kelch-like ECH-associated protein 1 (KEAP1), serine/threonine kinase 11, epidermal growth factor receptor, neurofibromin 1 (NF1), SET domain containing 2, RNA binding motif protein 10, MGA, MET, AT-rich interaction domain 1A, phosphatidylinositol-4,5-bisphosphate 3-kinase catalytic subunit $\alpha$ (PIK3CA), SWI/SNF-associated matrix-associated actin dependent regulator of chromatin subfamily A member 4, RB transcriptional corepressor 1 (RB1), cyclin dependent kinase inhibitor 2A (CDKN2A), U2 small nuclear RNA auxiliary factor 1, Ras-like without CAAX 1, Erb-B2 receptor tyrosine kinase 2 and ALK. For LUSC, data were obtained from the provisional dataset of 504 samples. Mutation data were obtained for TP53, CDKN2A, phosphatase and tensin homolog (PTEN), PIK3CA, KEAP1, major histocompatibility complex class I A, nuclear factor erythroid 2 like 2 (NFE2L2), NOTCH1 and RB1.

Statistical analysis. Median score and 5-95 percentile were plotted. Skp2 expression among different groups according to pathological grading, lymph node metastasis, smoking status and differentiation grade were evaluated by Kruskal-Wallis H-test which is a non-parametric method for testing whether samples originate from the same distribution. Wilcoxon matched-pairs signed rank test were used to compare Skp2 
Table I. Correlation between Skp2 protein expression and clinicopathological parameters in lung cancers.

\begin{tabular}{|c|c|c|c|c|c|c|c|c|c|c|c|}
\hline \multirow{3}{*}{$\begin{array}{l}\text { Histology } \\
\text { Clinicopathological } \\
\text { parameters }\end{array}$} & \multicolumn{6}{|c|}{ LUAD } & \multicolumn{5}{|c|}{ LUSC } \\
\hline & \multirow[b]{2}{*}{ Category } & \multirow[b]{2}{*}{ Cases } & \multicolumn{2}{|c|}{$\begin{array}{c}\text { Skp2 } \\
\text { expression }\end{array}$} & \multirow{2}{*}{$\begin{array}{l}\text { Statistical } \\
\text { value }\end{array}$} & \multirow[b]{2}{*}{ P-value } & \multirow[b]{2}{*}{ Cases } & \multicolumn{2}{|c|}{$\begin{array}{c}\text { Skp2 } \\
\text { expression }\end{array}$} & \multirow{2}{*}{$\begin{array}{l}\text { Statistical } \\
\text { value }\end{array}$} & \multirow[b]{2}{*}{ P-value } \\
\hline & & & Low & High & & & & Low & High & & \\
\hline Total & & 351 & 231 & 120 & & & 149 & 54 & 95 & & \\
\hline \multirow[t]{2}{*}{ Sex } & Male & 172 & 102 & 70 & $6.325^{\mathrm{b}}$ & $0.012^{\mathrm{a}}$ & 133 & 48 & 85 & $0.012^{\mathrm{b}}$ & 0.912 \\
\hline & Female & 179 & 129 & 50 & & & 16 & 6 & 10 & & \\
\hline \multirow[t]{2}{*}{ Age } & $<65$ & 186 & 129 & 57 & $2.207^{\mathrm{b}}$ & 0.137 & 81 & 34 & 47 & $2.525^{\mathrm{b}}$ & 0.112 \\
\hline & $\geq 65$ & 165 & 102 & 63 & & & 68 & 20 & 48 & & \\
\hline \multirow[t]{3}{*}{ Differentiation } & Well & 107 & 91 & 16 & $36.308^{\mathrm{c}}$ & $<0.001^{\mathrm{a}}$ & 11 & 7 & 4 & $2.898^{\mathrm{c}}$ & 0.235 \\
\hline & Moderate & 122 & 78 & 44 & & & 68 & 25 & 43 & & \\
\hline & Poor & 122 & 62 & 60 & & & 70 & 22 & 48 & & \\
\hline Smoking status & 0 & 232 & 163 & 69 & $16.198^{c}$ & $<0.001^{\mathrm{a}}$ & 31 & 14 & 17 & $6.555^{\mathrm{c}}$ & $0.038^{\mathrm{a}}$ \\
\hline \multirow[t]{2}{*}{ (cigarettes/year) } & $0<\mathrm{n} \leq 400$ & 62 & 41 & 21 & & & 30 & 7 & 23 & & \\
\hline & $>400$ & 57 & 27 & 30 & & & 88 & 33 & 55 & & \\
\hline \multirow[t]{4}{*}{$\mathrm{T}$} & 1 & 157 & 110 & 47 & $4.410^{c}$ & 0.221 & 30 & 9 & 21 & $0.653^{\mathrm{c}}$ & 0.884 \\
\hline & 2 & 170 & 110 & 60 & & & 86 & 35 & 51 & & \\
\hline & 3 & 13 & 6 & 7 & & & 26 & 8 & 18 & & \\
\hline & 4 & 11 & 5 & 6 & & & 7 & 2 & 5 & & \\
\hline \multirow[t]{4}{*}{$\mathrm{N}$} & 0 & 233 & 174 & 59 & $24.081^{\mathrm{c}}$ & $<0.001^{\mathrm{a}}$ & 72 & 23 & 49 & $2.564^{\mathrm{c}}$ & 0.464 \\
\hline & 1 & 35 & 17 & 18 & & & 33 & 13 & 20 & & \\
\hline & 2 & 79 & 37 & 42 & & & 43 & 17 & 26 & & \\
\hline & $\mathrm{x}$ & 4 & 3 & 1 & & & 1 & 1 & 0 & & \\
\hline \multirow[t]{2}{*}{ M } & 0 & 334 & 219 & 115 & $0.181^{\mathrm{b}}$ & 0.670 & 146 & 52 & 94 & $1.227^{\mathrm{b}}$ & 0.268 \\
\hline & 1 & 17 & 12 & 5 & & & 3 & 2 & 1 & & \\
\hline \multirow[t]{4}{*}{ TNM stage } & I & 215 & 165 & 50 & $33.202^{\mathrm{c}}$ & $<0.001^{\mathrm{a}}$ & 54 & 19 & 35 & $1.127^{\mathrm{c}}$ & 0.771 \\
\hline & II & 44 & 19 & 25 & & & 41 & 14 & 27 & & \\
\hline & III & 75 & 35 & 40 & & & 51 & 19 & 32 & & \\
\hline & IV & 17 & 12 & 5 & & & 3 & 2 & 1 & & \\
\hline \multirow[t]{2}{*}{ Diameter } & $\leq 3 \mathrm{~cm}$ & 270 & 195 & 75 & $21.368^{\mathrm{b}}$ & $<0.001^{\mathrm{a}}$ & 47 & 15 & 32 & $0.556^{\mathrm{b}}$ & 0.456 \\
\hline & $>3 \mathrm{~cm}$ & 81 & 36 & 45 & & & 102 & 39 & 63 & & \\
\hline
\end{tabular}

${ }^{\mathrm{a}} \mathrm{P}<0.05$. ' $\mathrm{Z}$-value from Kruskal-Wallis H-test. ${ }^{\mathrm{c}} \chi^{2}$ value from Pearson's $\chi^{2}$ test. Skp2, S-phase kinase associated protein 2 ; TNM, tumor-node-metastasis; LUAD, lung adenocarcinoma; LUSC, lung squamous cell carcinoma; $\mathrm{x}$, lymph node metastasis could not be assessed.

expression in LUAD and LUSC Pearson's $\chi^{2}$ was used to compare Skp2 expression in other clinical features, including histological, age, sex, tumor size and distant metastasis. Disease-free survival (DFS) was defined as the period between surgery and the date of recurrence, and overall survival (OS) was considered as the interval from surgery to the date of mortality or final follow-up. Kaplan-Meier method with the log-rank test was performed to determine the difference in survival between groups. Cox proportional hazards regression analysis was performed to estimate the hazard ratios for positive risk factors with the backward conditional elimination method (24). $\mathrm{P}<0.05$ was considered to indicate a statistically significant difference (two sided). SPSS 22.0 statistic software (IBM Corp., Armonk, NY, USA) and GraphPad Prism 6 software (GraphPad Software, Inc., La Jolla, CA, USA) were used to perform the computation for all statistical analyses.

\section{Results}

Correlation between Skp2 protein expression assessed by TMA-IHC and clinicopathological parameters in LUSC and $L U A D$. Skp2 protein expression was assessed by TMA-IHC analysis in the primary lung cancer tissues. Skp2 staining was primarily localized in the nuclear area, which is consistent with a previous study (2) (Fig. 1). Another previous study indicated that Skp2 expression was significantly higher in LUSC, compared with LUAD, which was verified in the present study $(\mathrm{P}<0.0001$; Fig. $1 \mathrm{~A}$ and $\mathrm{B})$. The median score of Skp2 in LUAD and LUSC were 0.00 and 0.375 , respectively. In NSCLC (LUAD and LUSC), the 25th, 50th, 75th quartiles of Skp2 expression final score were 0.000, 0.025 and 0.250. Distribution of the Skp2 expression score in the three intervals were notably different. The majority of Skp2 expression scores 
A

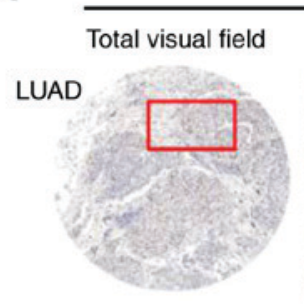

Cancer tissue
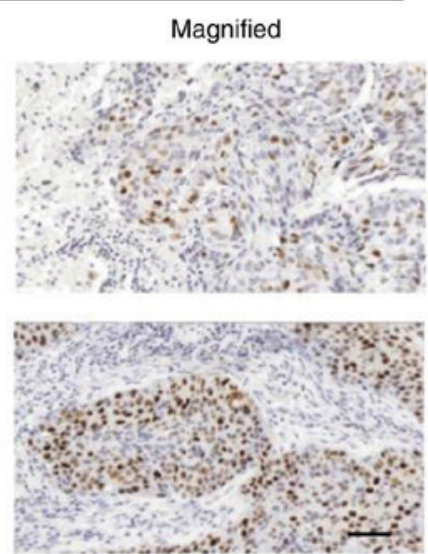

B

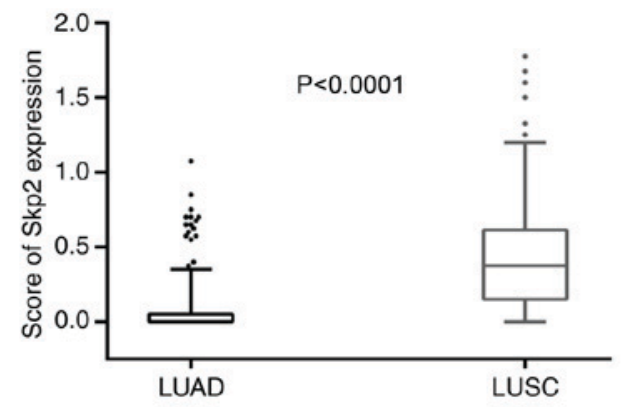

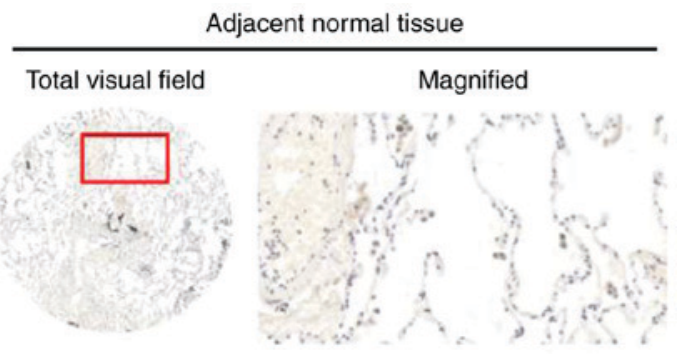
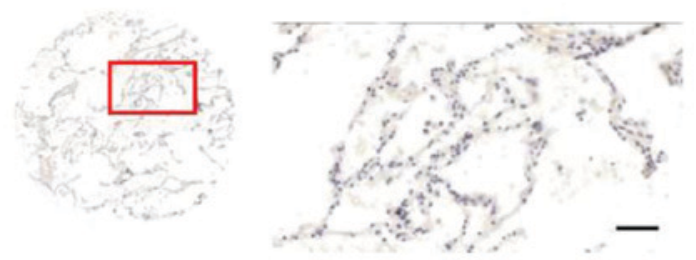

C

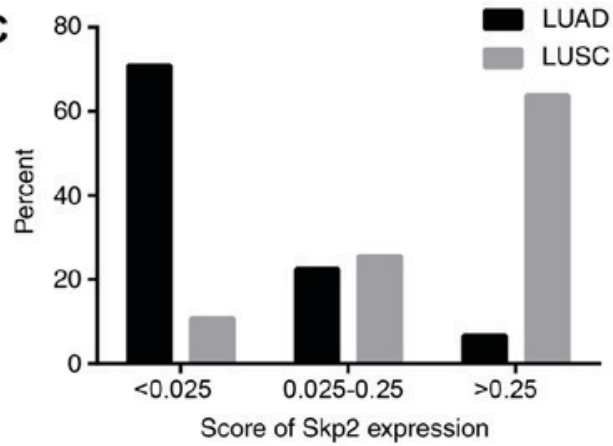

Figure 1. Skp2 expression in lung cancers. (A) Immunohistochemical staining of Skp2 in LUAD, LUSC and adjacent normal tissue (magnification, x100). Red rectangles indicate that the areas been magnified. Scale bars, $100 \mu \mathrm{m}$. (B) Boxplot of Skp2 expression scores in LUAD and LUSC. (C) Distribution of Skp2 expression score in the four intervals based on the 25th, 50th and 75th percentile in non-small cell lung cancer. LUAD, lung adenocarcinoma; LUSC, lung squamous cell carcinoma; Skp2, S-phase kinase associated protein 2.

were $<0.025$ in the LUAD group, while scores $>0.250$ were observed more frequently in LUSC (Fig. 1C). Based on these data, subgroups analysis was proceeded in LUAD and LUSC, separately. A score of 0.025 was used to define high expression and low expression in LUAD, and a score of 0.250 in LUSC was used identically.

In LUAD, Skp2 expression varied in different groups according to sex, differentiation, smoking history, TumorNode-Metastasis (TNM) stage (25), lymph node metastasis and tumor diameter $(\mathrm{P}<0.05$; Table I). In LUSC, Skp2 protein expression correlated with smoking status $(\mathrm{P}<0.05$; Table I), whilst it demonstrated limited correlation with other clinical features.

Prognostic significance of Skp2 protein expression in LUAD. There were three patients who succumbed during the follow-up, shortly following leaving the hospital. Survival analyses were performed in 348 patients with LUAD, of which 120 patients had a high expression of Skp2 and 228 patients had a low expression. Kaplan-Meier survival analysis with log-rank test indicated that a high Skp2 protein expression in patients with LUAD was significantly associated with a reduced DFS and OS ( $\mathrm{P}<0.001$; Fig. 2A and B). The 5-year OS in the Skp2-H group was $61.9 \%$, compared with a rate of $86.9 \%$ in the Skp2-L group. For patients with LUSC, no association was observed between Skp2 expression and the outcome of patients (Fig. 2C and D).

Cox proportional hazards model was used to determine the association of six factors (sex, age, differentiation, pathological stage, smoking status and Skp2 protein expression) with the OS of patients with LUAD. As a result, Skp2 protein highly expression was demonstrated to be an independent prognostic factor of OS, with a relative risk of $1.845(\mathrm{P}=0.030)$. Poor differentiation $(\mathrm{P}=0.016)$ and high stage $(\mathrm{P}<0.001)$ were associated with a reduced OS also (Table II).

Histological differential mRNAs and frequent mutations association with $S k p 2$. Association analysis and cluster analysis screened nine Skp2 co-expression mRNAs [cell division cycle 45 (CDC45), replication factor C subunit 4 (RFC4), actin like 6A (ACTL6A), family with sequence similarity 83 member D, E2F transcription factor 7, chromosome 15 open reading frame 42 (C15orf42), DNA polymerase $\theta$ (POLQ), Skp2 and minichromosome maintenance complex component 2 (MCM2)], which are also differential between LUAD and LUSC. Skp2, CDC45 and MCM2 are involved in the $\mathrm{G}_{1} / \mathrm{S}$ transition of the mitotic cell cycle. CDC45, MCM2, RFC4, POLQ and ACTL6A participate in DNA replication and repair (Fig. 3).

In the TCGA cohort of patients with LUAD and LUSC, Skp2 expression was highly expressed in the TP53, NF1 and RB1 mutation groups, compared with the unaltered group. The KRAS mutation group had a lower Skp2 expression in LUAD. Whilst in LUSC, it was determined that there was a significant deviation of Skp2 expression in the four other commonly mutated genes, PTEN, PIK3CA, KEAP1 and NFE2L2 (Table III). 
Table II. Multivariate analysis for prognostic factors in patients with lung adenocarcinoma.

OS

\begin{tabular}{lccccc}
\cline { 2 - 6 } Variable & Regression coefficient & S.E. & Relative risk & 95\%CI & P-value \\
\hline Sex (male vs. female) & -0.221 & 0.273 & 0.801 & $0.469-1.368$ & 0.417 \\
Age (<65 vs. $\geq 65)$ & 0.536 & 0.277 & 1.709 & $0.993-2.941$ & 0.053 \\
Differentiation (well/moderate vs. poor) & 1.282 & 0.531 & 3.604 & $1.272-10.214$ & $0.016^{\mathrm{a}}$ \\
TNM stage (stage I vs. stages II-IV) & 1.484 & 0.324 & 4.413 & $2.340-8.320$ & $<0.001^{\mathrm{a}}$ \\
Smoking status (non-smoker vs. smoker) & -0.322 & 0.354 & 0.752 & $0.362-1.452$ & 0.364 \\
Skp2 expression (low vs. high) & 0.613 & 0.283 & 1.845 & $1.060-3.212$ & $0.030^{\mathrm{a}}$ \\
\hline
\end{tabular}

${ }^{\mathrm{a}} \mathrm{P}<0.05$. ${ }^{\mathrm{b}}$ Never smoked. TNM, tumor-node-metastasis; CI, confidence interval; S.E., standard error; OS, overall survival; Skp2, S-phase kinase associated protein 2 .

A

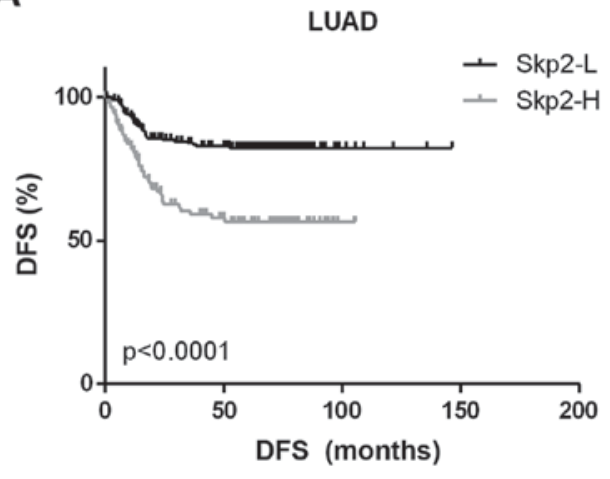

C

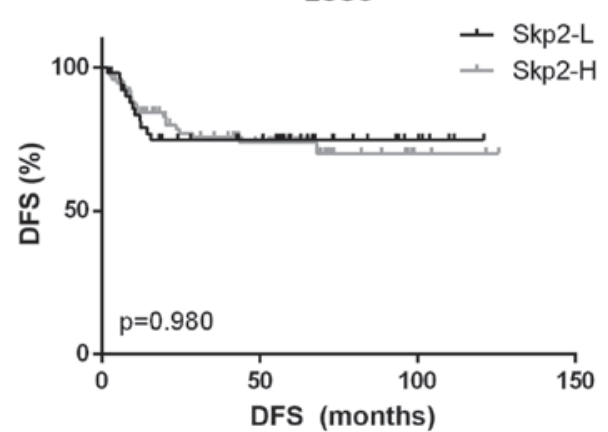

B

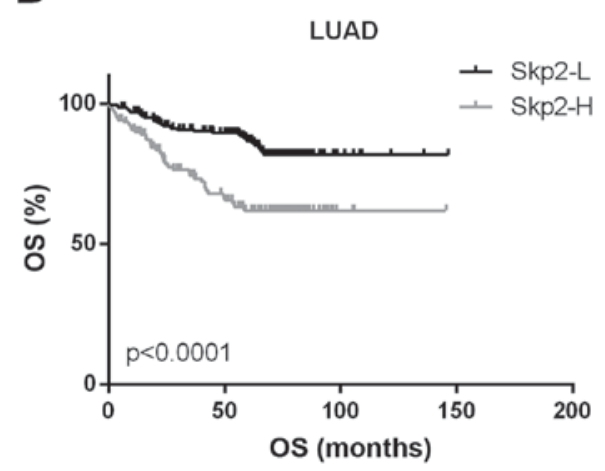

D

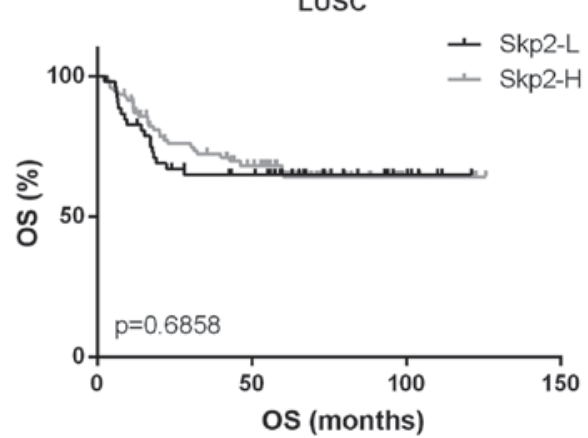

Figure 2. Kaplan-Meier survival analysis for patients with LUAD and LUSC with Skp2-H and Skp2-L. (A) DFS in patients with LUAD. (B) OS in patients with LUAD. (C) DFS in patients with LUSC. (D) OS in patients with LUSC. Skp2-L, Skp2 low expression; Skp2-H, Skp2 high expression; OS, overall survival; DFS, disease-free survival; Skp2, S-phase kinase associated protein 2; LUAD, lung adenocarcinoma; LUSC, lung squamous cell carcinoma.

\section{Discussion}

LUAD and LUSC are the NSCLC types with the highest prevalence, accounting for $80-85 \%$ of lung cancer types. Large-scale sequencing studies have revealed the genomic differences between LUAD and LUSC (26). The molecular mechanisms underlying LUAD and LUSC are considerably different (27). A previous study demonstrated that the Skp2 relative gene copy number aberrations were detected in NSCLC, and these changes are reflected at the mRNA and protein expression levels (16).

In the present study, Skp2 protein has different expression patterns in LUSC and LUAD; therefore the association between
Skp2 expression and clinicopathological parameters in patients with LUAD and LUSC were evaluated. The data indicated that clinicopathological and prognostic implications based on Skp2 expression in LUAD and LUSC should be considered different.

The data indicated that high expression of Skp2 in LUSC would result in abnormal activation of DNA replication and $\mathrm{G}_{1} / \mathrm{S}$ transition pathways. This indicated that alterations of lung cancer associated oncogenes modulating these pathways may contribute to the aberration of Skp2 expression. The association between lung cancer mutations and Skp2 expression were then investigated. Skp2 was expressed higher in the lung cancer-associated genes altered group, except in the KRAS 
Table III. Deviation of S-phase kinase associated protein 2 expression with lung cancer mutations.

\begin{tabular}{|c|c|c|c|c|c|c|c|}
\hline \multirow[b]{2}{*}{ Histology } & \multirow[b]{2}{*}{ Gene $(\mathrm{n} / \%)$} & \multicolumn{2}{|c|}{ Mean } & \multicolumn{2}{|c|}{$\mathrm{SD}$} & \multirow[b]{2}{*}{ P-value } & \multirow[b]{2}{*}{ Q-value } \\
\hline & & Altered group & Unaltered group & Altered group & Unaltered group & & \\
\hline \multirow[t]{4}{*}{ LUAD } & TP53 (111/21.3\%) & 7.44 & 7.05 & 1.08 & 0.97 & $9.270 \times 10^{-4}$ & $7.036 \times 10^{-3}$ \\
\hline & KRAS $(103 / 19.7 \%)$ & 6.90 & 7.20 & 0.79 & 1.04 & $1.639 \times 10^{-3}$ & 0.0324 \\
\hline & NF1 $(29 / 13 \%)$ & 7.61 & 7.10 & 1.02 & 1.00 & 0.0102 & 0.193 \\
\hline & RB1 $(17 / 7.4 \%)$ & 7.98 & 7.10 & 1.00 & 0.99 & $3.696 \times 10^{-4}$ & 0.0178 \\
\hline \multirow[t]{6}{*}{ LUSC } & TP53 (150/29.8\%) & 9.22 & 8.79 & 0.83 & 0.96 & $6.32 \times 10^{-7}$ & $6.708 \times 10^{-6}$ \\
\hline & PTEN (74/14.7\%) & 9.24 & 8.87 & 0.86 & 0.95 & $1.091 \times 10^{-3}$ & 0.119 \\
\hline & PIK3CA (251/49.8) & 9.06 & 8.77 & 0.83 & 1.03 & $5.430 \times 10^{-4}$ & $2.352 \times 10^{-3}$ \\
\hline & KEAP1 (40/7.9\%) & 9.28 & 8.89 & 0.90 & 0.94 & 0.0104 & 0.0869 \\
\hline & NFE2L2 (58/11.5\%) & 9.16 & 8.88 & 0.88 & 0.95 & $7.959 \times 10^{-3}$ & 0.0348 \\
\hline & RB1 $(33 / 6.5 \%)$ & 9.39 & 8.88 & 0.90 & 0.94 & $1.199 \times 10^{-3}$ & 0.0394 \\
\hline
\end{tabular}

SD, standard deviation; LUAD, lung adenocarcinoma; LUSC, lung squamous cell carcinoma; TP53, tumor protein P53; NF1, neurofibromin 1; RB1, RB transcriptional corepressor 1; PTEN, phosphatase and tensin homolog; PIK3CA, phosphatidylinositol-4,5-bisphosphate 3-kinase catalytic subunit $\alpha$; KEAP1; Kelch-like ECH-associated protein 1; NFE2L2, nuclear factor erythroid 2 like 2.

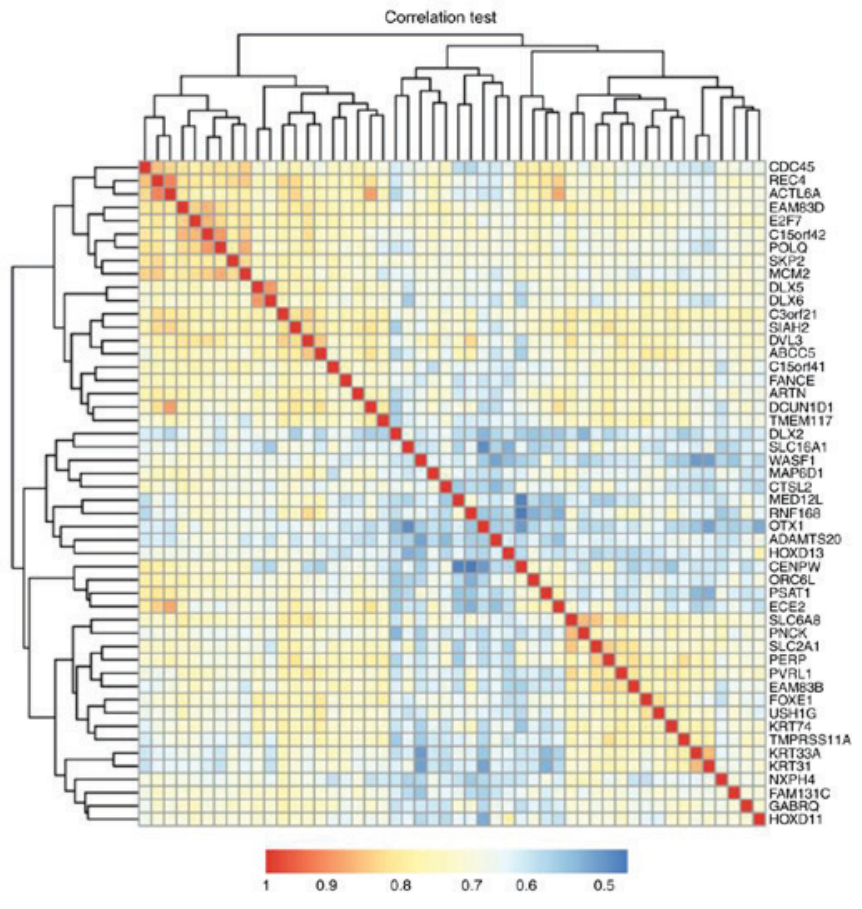

Figure 3. Heatmap with clustering analysis of the top 50 S-phase kinase associated protein 2 co-expressed mRNAs, which were differential between lung adenocarcinoma and lung squamous cell carcinoma.

mutation group. KRAS has a mutation rate of $19.7 \%$ in LUAD, which is more frequent than LUSC; however, PTEN, PIK3CA, KEAP1 and NFE2L2, which induce the proliferation of cancer cells, are present more frequently in LUSC. Different driver gene mutation frequencies may be one reason for the distinct expression patterns of Skp2 in LUSC and LUAD; however, this would not fully explain the difference of Skp2 expression between LUAD and LUSC. Further study focusing on the regulation of Skp2 expression are required, including the regulated relationship with deviated miRNAs and lncRNAs.
Skp2 has a function in promoting cell growth (28). A previous study primarily focused on the T stage (29). In the present study, Skp2 overexpression was associated with the tumor diameter more significantly than $\mathrm{T}$ stage $(\mathrm{P}<0.001$ vs. $\mathrm{P}=0.221$; Table I). Ina ddition to the $\mathrm{T}$ stage, continued studies must also consider pleural invasion and the distance to the carina of the trachea, but these features are not affected by tumor proliferation ability.

The prognostic value of Skp2 expression in NSCLC has been controversial. Osoegawa et al (1) indicated that a high Skp2 protein expression was an independent poor prognostic marker in NSCLC; however, Zhu et al (30) reported that Skp2 overexpression was not prognostically significant alone, but along with a RAS mutation was a significant independent poor prognostic marker in NSCLC. In the present study, it was demonstrated that a high expression of $\mathrm{Skp} 2$ may indicate a poor prognosis in LUAD, but it was not observed in LUSC. Additionally, in KRAS-mutated LUAD, Skp2 had a lower expression level, compared with the unaltered group. This may indicate that Skp2 may have clinicopathological and prognostic implications when it is expressed at a relatively lower level.

Detection of Skp2 overexpression may be a beneficial marker for prognosis. Skp2 may provide sputum-based markers that have the potential to improve the early detection of LUSC (31). Skp2 can also be detected in the peripheral blood of patients with NSCLC, and has demonstrated high diagnostic value (32). These studies provide evidence that detection of Skp2 expression level should be conducted prior to surgery, assisting with the decision for surgery procedure; however, the molecular mechanism underlying regulation of Skp2 in lung cancer remains unclear and further in vitro and in vivo investigations are required.

To conclude, Skp2 protein expression has different patterns between LUAD and LUSC. The clinicopathological and prognostic implications based on Skp2 expression in LUAD and LUSC should be considered different. Differential expression 
of Skp2 protein expression was associated with an unfavorable outcome in patients with LUAD, but not in LUSC.Skp2-HLUSC may have robust proliferation ability.

\section{Acknowledgements}

Not applicable.

\section{Funding}

The present study was funded by the National High Technology Research and Development Program of China (863 Program) (grant nos. ss2014AA020602 and ss2014AA020604).

\section{Availability of data and materials}

The datasets used and/or analyzed during the current study are available from the corresponding author on reasonable request.

\section{Authors' contributions}

$\mathrm{KZ}$ analyzed and interpreted the data regarding the Skp2 expression and clinicopathological parameters, and also was a major contributor in writing the manuscript. QH and JC performed the tissue microarray and immunohistochemical analyze. FY and JW made substantial contributions to conception and design. All authors read and approved the final manuscript.

\section{Ethics approval and consent to participate}

All procedures performed in the current study involving human participants were in accordance with the ethical standards of the Ethics Committee of Peking University People's Hospital and with the 1964 Helsinki declaration and its later amendments or comparable ethical standards. Informed consent was obtained from all individual participants included in the study.

\section{Consent for publication}

Written informed consent for the publication of any associated data and accompanying images was obtained from all the patient, or parent, guardian or next of kin (in case of deceased patients).

\section{Competing interests}

The authors declare that they have no competing interests.

\section{References}

1. Osoegawa A, Yoshino I, Tanaka S, Sugio K, Kameyama T, Yamaguchi $\mathrm{M}$ and Maehara Y: Regulation of p27 by S-phase kinase-associated protein 2 is associated with aggressiveness in non-small-cell lung cancer. J Clin Oncol 22: 4165-4173, 2004.

2. Gstaiger M, Jordan R, Lim M, Catzavelos C, Mestan J, Slingerland J and Krek W: Skp2 is oncogenic and overexpressed in human cancers. Proc Natl Acad Sci USA 98: 5043-5048, 2001.

3. Nakayama KI, Hatakeyama S and Nakayama K: Regulation of the cell cycle at the G1-S transition by proteolysis of cyclin E and p27Kip1. Biochem Biophys Res Commun 282: 853-860, 2001.
4. Nakayama K, Nagahama H, Minamishima YA, Matsumoto M, Nakamichi I, Kitagawa K, Shirane M, Tsunematsu R, Tsukiyama T, Ishida $\mathrm{N}$, et al: Targeted disruption of $\mathrm{Skp} 2$ results in accumulation of cyclin E and p27(Kip1), polyploidy and centrosome overduplication. EMBO J 19: 2069-2081, 2000.

5. Sicari BM, Troxell R, Salim F, Tanwir M, Takane KK and Fiaschi-Taesch N: c-myc and skp2 coordinate p27 degradation, vascular smooth muscle proliferation, and neointima formation induced by the parathyroid hormone-related protein. Endocrinology 153: 861-872, 2012.

6. Suzuki S, Fukasawa H, Misaki T, Togawa A, Ohashi N, Kitagawa K, Kotake Y, Liu N, Niida H, Nakayama K, et al: The amelioration of renal damage in Skp2-deficient mice canceled by p27 Kip1 deficiency in Skp2 $2^{-/-}$p2 $7^{-/-}$mice. PLoS One 7: e36249, 2012.

7. Lim MS, Adamson A, Lin Z, Perez-Ordonez B, Jordan RC, Tripp S, Perkins SL and Elenitoba-Johnson KS: Expression of Skp2, a p27(Kip1) ubiquitin ligase, in malignant lymphoma: Correlation with p27(Kip1) and proliferation index. Blood 100: 2950-2956, 2002.

8. Wang Z, Gao D, Fukushima H, Inuzuka H, Liu P, Wan L, Sarkar FH and Wei W: Skp2: A novel potential therapeutic target for prostate cancer. Biochim Biophys Acta 1825: 11-17, 2012.

9. Rose AE, Wang G, Hanniford D, Monni S, Tu T, Shapiro RL, Berman RS, Pavlick AC, Pagano M, Darvishian F, et al: Clinical relevance of SKP2 alterations in metastatic melanoma. Pigment Cell Melanoma Res 24: 197-206, 2011.

10. Schüler S, Diersch S, Hamacher R, Schmid RM, Saur D and Schneider G: SKP2 confers resistance of pancreatic cancer cells towards TRAIL-induced apoptosis. Int J Oncol 38: 219-225, 2011.

11. Osoegawa A, Yoshino I, Tanaka S, Sugio K, Kameyama T, Yamaguchi $\mathrm{M}$ and Maehara Y: Regulation of p27 by S-phase kinase-associated protein 2 is associated with aggressiveness in non-small-cell lung cancer. J Cinical Oncol 22: 4165-4173, 2004.

12. Wang Z, Fukushima H, Inuzuka H, Wan L, Liu P, Gao D, Sarkar FH and Wei W: Skp2 is a promising therapeutic target in breast cancer. Front Oncol 1: 18702, 2012.

13. Tian YF, Chen TJ, Lin CY, Chen LT, Lin LC, Hsing CH, Lee SW, Sheu MJ, Lee HH, Shiue YL, et al: SKP2 overexpression is associated with a poor prognosis of rectal cancer treated with chemoradiotherapy and represents a therapeutic target with high potential. Tumor Biol 34: 1107-1117, 2013.

14. Hershko DD: Oncogenic properties and prognostic implications of the ubiquitin ligase Skp2 in cancer. Cancer 112: 1415-1424, 2008.

15. Timmerbeul I, Garrett-Engele CM, Kossatz U, Chen X, Firpo E, Grünwald V, Kamino K, Wilkens L, Lehmann U, Buer J, et al: Testing the importance of p27 degradation by the SCFskp2 pathway in murine models of lung and colon cancer. Proc Natl Acad Sci USA 103: 14009-14014, 2006.

16. Yokoi S, Yasui K, Mori M, Iizasa T, Fujisawa T and Inazawa J: Amplification and overexpression of SKP2 are associated with metastasis of non-small-cell lung cancers to lymph nodes. Am J Pathol 165: 175-180, 2004.

17. Hewitt SM: Tissue microarrays as a tool in the discovery and validation of predictive biomarkers. Methods Mol Biol 823: 201-214, 2012.

18. Remmele W and Stegner HE: Recommendation for uniform definition of an immunoreactive score (IRS) for immunohistochemical estrogen receptor detection (ER-ICA) in breast cancer tissue. Pathologe 8: 138-140, 1987 (In German).

19. Robinson MD and Oshlack A: A scaling normalization method for differential expression analysis of RNA-seq data. Genome Biol 11: R25, 2010.

20. Huang da W, Sherman BT and Lempicki RA: Systematic and integrative analysis of large gene lists using DAVID bioinformatics resources. Nat Protoc 4: 44-57, 2009.

21. Huang da W, Sherman BT and Lempicki RA: Bioinformatics enrichment tools: Paths toward the comprehensive functional analysis of large gene lists. Nucleic Acids Res 37: 1-13, 2009.

22. Gao J, Aksoy BA, Dogrusoz U, Dresdner G, Gross B, Sumer SO, Sun Y, Jacobsen A, Sinha R, Larsson E, et al: Integrative analysis of complex cancer genomics and clinical profiles using the cBioPortal. Sci Signal 6: pl1, 2013.

23. Cerami E, Gao J, Dogrusoz U, Gross BE, Sumer SO, Aksoy BA, Jacobsen A, Byrne CJ, Heuer ML, Larsson E, et al: The cBio cancer genomics portal: An open platform for exploring multidimensional cancer genomics data. Cancer Discov 2: 401-404, 2012. 
24. Costacou T, Lopes-Virella MF, Zgibor JC, Virella G, Otvos J, Walsh M and Orchard TJ: Markers of endothelial dysfunction in the prediction of coronary artery disease in type 1 diabetes. The Pittsburgh epidemiology of diabetes complications study. J Diabetes Complications 19: 183-193, 2005.

25. Edge SB and Compton CC: The American Joint Committee on Cancer: The 7th edition of the AJCC cancer staging manual and the future of TNM. Ann Surg Oncol 17: 1471-1474, 2010.

26. Campbell JD, Alexandrov A, Kim J, Wala J, Berger $\mathrm{AH}$ Pedamallu CS, Shukla SA, Guo G, Brooks AN, Murray BA, et al: Distinct patterns of somatic genome alterations in lung adenocarcinomas and squamous cell carcinomas. Nat Genet 48: 607-616, 2016.

27. Liu J, Yang XY and Shi WJ: Identifying differentially expressed genes and pathways in two types of non-small cell lung cancer: Adenocarcinoma and squamous cell carcinoma. Genet Mol Res 13: 95-102, 2014.

28. Su L, Han D, Wu J and Huo X: Skp2 regulates non-small cell lung cancer cell growth by Meg3 and miR-3163. Tumour Biol 37: 3925-3931, 2016

29. Takanami I: The prognostic value of overexpression of Skp2 mRNA in non-small cell lung cancer. Oncol Rep 13: 727-731, 2005 .
30. Zhu CQ, Blackhall FH, Pintilie M, Iyengar P, Liu N, Ho J, Chomiak T, Lau D, Winton T, Shepherd FA and Tsao MS.: Skp2 gene copy number aberrations are common in non-small cell lung carcinoma, and its overexpression in tumors with ras mutation is a poor prognostic marker. Clin Cancer Res 10: 1984-1991, 2004.

31. Jiang F, Todd NW, Li R, Zhang H, Fang H and Stass SA: A panel of sputum-based genomic marker for early detection of lung cancer. Cancer Prev Res (Phila) 3: 1571-1578, 2010.

32. Chiang IT, Wang WS, Liu HC, Yang ST, Tang NY and Chung JG: Curcumin alters gene expression-associated DNA damage, cell cycle, cell survival and cell migration and invasion in NCI-H460 human lung cancer cells in vitro. Oncology Rep 34: 1853-1874, 2015. 